als bei der typischen Form; die Blumenblïtter haben aber am Grund den orangegelben Fleck. - Diese an lichten Stellen der Föhrenwälder in Bernstein sehr verbreitete Varietät ist unter den Variationen der $P$. serpentini Börb. die auffälligste, denn sowohl die typische Form, als auch die var. parvifrons Borb. bildet mit ihren zahlreichen $10-25 \mathrm{~cm}$ langen Stengeln dichte Rasen und trägt grössere Blüten. - Zugleich will ich hier bemerken, dass die Blumenblätter der P. Serpentini Borb. in allen ihren Formen am Grunde einen mehr minder ausgeprägten orangegelben Fleck aufweisen, welches charakteristische Merkmal nebst anderen Kennzeichen auf ihre nahe Verwandtschaft mit $P$. salisburgensis Haenke, als welche ich diese Pflanze seinerzeit (1883) einlegte, hinweiset.

P. pseudo-serpentini m. P. serpentini Borb. $\times$ glandulifera Kras (vergl. Oest. botan. Zeitschr. 1893, pag. 357). Auf Serpentin in Bernstein. - Die in Bernstein gesammelten Exemplare stehen zum Theil der $P$. serpentini näher, manche sind blos durch die Drüsenhaare an dem oberen Theile der Stengel und an den Blütenstielen, ferner durch kleinere Blüten von dieser zu unterscheiden. Die der P. glaudulifera näher stehenden Formen weichen von dieser ab ausser durch zahlreichere Blattzähne und subsessile Drüsenköpfchen an Stengel und Blättern, noch durch den orangegelben Fleck der Petalen und die mehr abstehenden Haare der Blattstiele.

Lotus tenuifolius L. var. major Scop. - Gräben in Güns.

\title{
Zopf W., Uebersicht der auf Flechten schmarotzenden Pilze.
}

Besprochen von Prof. E. Kernstock (Klagenfurt).

In der "Hedwigia ", Band XXXV (1896), Seite 312-366, hat Prof. Dr.Wilh. Z o p in Halle eine „Uebersicht der auf Flechten schmarotzenden Pilze" gegeben. Mit den letzten Worten präcisirt der Verfasser seinen Standpunkt in der Flechtenparasitenfrage und stellt sich hiermit an die Seite Rehm's und in Gegensatz zu Minks.

Es wäre sehr erfreulich, wenn der Herr Verfasser in seiner von ihm angezeigten Monographie dieser Schmarotzer (in den "Nova Acta. Acad. Leopold.") sich des Näheren über seine diesbezüglichen Ansichten aussprechen möchte. Was die Uebersicht anbelangt, so wird sie wegen ihrer Reichhaltigkeit und Uebersichtlichkeit von den Lichenologen freudigst begrüsst werden. Bei jeder der alphabetisch geordneten Flechtenarten verzeichnet der Verfasser die auf ihr beobachtéten pyrenocarpen und discocarpen Schmarotzer und Conidienfructificationen.

Jeder weniger allgemein bekannten oder neuen Parasitenspecies ist eine kurze Diagnose beigefügt, welche in den meisten Fällen der Absicht des Verfassers vortrefflich dienen wird. Zweifelsohne hat derselbe bei der Bearbeitung der seit vielen Jahren 
angesammelten Angaben über Flechtenparasiten eine kritische Sichtung, soweit sie möglich war, vorgenommen, und diese lief in manchen Fällen auf eine Reduction gewisser winziger Arten hinaus; nur wäre es dann vielleicht wünschenswerth gewesen, wenn dies an Ort und Stelle in der Vebersicht durch Anführung des oder der Synonyme kenntlich gemacht worden wäre. Wirkliche Vollständigkeit wird der Verfasser wohl nicht angestrebt haben; ich könnte aus meinem Handverzeichnisse eine ziemlich lange Reihe von Vorkommnissen anführen, welche in der Uebersicht nicht enthalten sind.

Meine eigenen kleinen Arbeiten über die Flechtenflora Steiermarks und Tirols enthaiten ebenfalls einige in der Uebersicht fehlende Beobachtungen, welche ich hiermit - der Herr Verfasser wird es mir nicht übel nehmen - zu seiner Verfügung stelle.

Es wurde beobachtet auf dem:

Thallus von Amphoridium Veronense: Tichothecium pygmaeum (Beitr. VII).

Thallus von Aspicilia calcarea: dasselbe (Beitr. VII). (Brixen).

Thallus von Aspicilia gibbosa: Xenosphaeria oligospora

Thallus von Biatora ochracea: Tichoth. pygmaeum (III).

Apoth.: Blastenia ferruginea: Cercidospora caudata n. sp. (VII).

'Thallus von Cladonia amaurocraea: Nesolechia punctum (Steiermark).

Thallus von Dermatocarpon pussillum : Leciogr. centrifuga var. conglobata. n. var. (VII).

Thallus von Diplotomma epipolium: Tichoth. gemmiferum (II).

Thallus von Gyalolechia lactea : Tichoth. pygmaeum (VII). (VI).

Apoth.: Gyalolechia lactea: Cercidospora caudata n. sp.

Thallus von Lecanora intricata: Tichoth. pygmaeum (St.).

Thallus von Lecanora polytropa: Nesolechia vitellinaria (VI). tula (VI).

Thallus von Lecanora polytropa: Nesolechia aggregan-

Thallus von Lecanora polytropa: Tichoth. pygmaeum v. grandiusculum (VI).

Apoth.: Lecanora polytropa: Conida subvarians (I), (VI).

Apoth.: Lecanora subfusca: dieselbe (III).

Thallus von Lecidea albocoerulescens: Tichoth. gemmiferum et pygmaeum (VI).

Thallus von Lecidea crustulata: Tichoth. pygmaeum (VI).

Thallus von Lecidea declinans: Tichoth. pygmaeum grandiusculum (St.) et calcaricolum (Brixen).

Thallus von Lecidea infidula: Tichoth. pygmaeum (IV).

Thallus von Lecidea latypea: dasselbe (VI). 
Thallus von Lecidea leucitica: dasselbe (Brixen, Steiermark).

Thallus von Lecidea lithyrga: dasselbe, rar. grandiuscuilum (Brixen).

Thallus ron Lecidea meiospora: Tichoth. gemmiferum (Brixen) et pygmaeum (VI).

Thallus von Lecidea petrosa: Tichoth. pygmaeum (IV).

Thallus von Lecidea vorticosa: Tichoth. pygnaeun grandiusculum (VI), Müllerella Zhallophila (VI).

Thallus von Parmelia exasperatula: Nesolechia oxyspora. (III).

Thallus von Parmelia prolixa: dieselbe (VII).

Thallus von Parmelia caesia: Tichoth. gemmiferum (VII).

Thallus von Parmelia saxatalis : Nesolechia punctum (VII).

Thallus von Peltidea aphthosa: Bilimbia sabulet. Killiasii (IV).

Thallus von Placodium saxicitum: Cercidospora epipolytropa (VI).

Thallus von Rinodina discolor $v$. candida: Tichoth. gemmiferum (VII).

Apoth. von Rinodina corticola : Tichoth. pygmaeum (III).

Apoth. von Rinodina metabolica: dasselbe (III).

Apoth. von Rinodina pyrina: dasselbe (III).

Apoth. von Scoliciosporum corticolum: Arthopyrenia conspurcans (VII).

Thallus von Vermucaria aquatilis: Arthopyrenia rivulorum (VII).

Klagenfurt, November 1896.

Ein Beitrag zur Pilz-Flora der Umgegend von Hohenstadt in Mähren.

\author{
Von Franz Bubak, \\ Gymnasialprofessor in Hohenstadt.
}

Seit 15. September l. J. weile ich in Hohenstadt und diesen Aufenthalt habe ich zur Erforschung der mykologischen Verhältnisse hiesiger Umgegend benützt. Obzwar es nur eine sehr kurze Zeit ist, die ich heuer meinem Zwecke widmen konnte, so gelang es mir doch, viele interessante Arten zu entdecken. Da mir die betreffende Literatur hier unzugänglich ist, kann ich nicht entscheiden, welche Species für Mähren neu sind.

Die grösste Aufmerksamkeit widmete ich den Uredineen und von diesen ist es mir gelungen, 52 Arten zu constatiren. Das Verzeichniss der Arten aus anderen Familien werde ich erst später zusammenstellen, jetzt veröffentliche ich nur die Ustilagineen und Uredineen. 